\title{
新型含氯代噻唑基团和嘧啶环的吡唑肜醚类衍生物的 合成及其生物活性研究
}

\author{
杨亚喆林大勇傅翠蓉邹小毛* \\ (南开大学元素有机化学研究所 元素有机化学国家重点实验室 天津化学化工协同创新中心 天津 300071)
}

\begin{abstract}
摘要 为解决抗性问题, 寻找新的活性化合物, 在前期工作基础上, 运用生物等排原理, 以吡唑肟梄为骨架, 引入了第 二代新烟碱类重要药效团氯代噻唑和另一重要基团嘧啶环, 设计合成了系列新型吡唑肜梄类化合物. 以 ${ }^{1} \mathrm{H} N \mathrm{NM}, \mathrm{IR}$, 元素分析及 MS 进行结构确证, 并进行了杀虫、杀螨及杀菌活性测定. 部分具有氯代噻夾环的 $\mathbf{A}$ 系列化合物显示了良 好的杀蚊虫活性 $\left(100 \%, 5 \mathrm{mg} \cdot \mathrm{L}^{-1}\right)$ 、杀豆蜴活性 $(100 \%, 200 \mathrm{mg} / \mathrm{L})$ 和杀螨活性 $(100 \%, 200 \mathrm{mg} / \mathrm{L})$; 具有嘧啶环的 B 系列化 合物显示了一定的杀菌活性.
\end{abstract}

关键词＼cjkstart吡唑肜醚; 氯代噻唑; 嘧啶环; 合成; 生物活性

\section{Synthesis and Biological Activity Evaluation of Novel Pyrazole Oxime Ether Derivatives Containing Chlorothiazole Group and Pyrimidine Rings}

\author{
Yang, Yazhe Lin, Dayong Fu, Cuirong Zou, Xiaomao* \\ (State Key Laboratory of Elemento-Organic Chemistry, Collaborative Innovation Center of Chemical Science and Engineering \\ (Tianjin), Institute of Elemento-Organic Chemistry, Nankai University, Tianjin 300071)
}

\begin{abstract}
On the basis of our previous work, in order to seeking for novel compounds with bioactivities to settle for the resistance problem, two series of novel pyrazole oxime ether derivatives have been designed and synthesized containing chlorothiazole group and pyrimidine rings applying the bioisosterism. Their structures were determined by ${ }^{1} \mathrm{H} \mathrm{NMR}$, IR, elemental analysis and MS, and their bioactivities were performed. Nearly half of the target compounds of series A with chlorothiazole group exhibited good insecticidal activity against culex pipiens pallens $\left(100 \%, 5 \mathrm{mg} \bullet \mathrm{L}^{-1}\right)$, several compounds of series $\mathbf{A}$ showed good activity against Aphis laburni Kaltenbach (100\%, $200 \mathrm{mg} / \mathrm{L})$ and Tetranychus cinnabarinus (100\%, $200 \mathrm{mg} / \mathrm{L})$, and the compounds of series $\mathbf{B}$ with pyrimidine rings exhibited certain fungicidal activity.
\end{abstract}

Keywords pyrazole oxime; chlorothiazole; pyrimidine rings; synthesis; bioactivity

吡唑肜醚类化合物是一类具有优良生物活性的化 合物，不仅在医药上有着广泛的应用，在农药上也是一 类重要的活性化合物, 广泛应用于杀虫剂、杀螨剂、杀 菌剂和除草剂等方面 ${ }^{[1 \sim 3]}$. 近年来出现的商品化的代表 有唑螨酯(fenpyroximate, 图 1) ${ }^{[4,5]}$. 有关它的作用机理 研究表明，有别于鱼藤酮等天然呼吸抑制剂，唑螨酯属 于化学合成的 NADH-泛醌氧化还原酶(Complex I)抑制 剂 ${ }^{[6]}$, 可以抑制作用对象的呼吸传递链中电子的转移, 具有靶标特征 ${ }^{[7]}$. 随着唑螨酯的广泛应用, 逐渐产生了
抗性问题 ${ }^{[8]}$. 因此, 为发现新活性化合物, 以唑螨酯的 吡唑朊醚骨架为中心, 针对它的 C 部分(图 1), 农药工作 者引入了各种活性基团 ${ }^{[9,10]}$, 如第一代新烟碱类杀虫剂 的活性基团氯代吡啶等 ${ }^{[11]}$. 在众多新化合物中, 其中不 乏具有高杀虫、杀螨和杀菌活性的化合物. 本课题组前 期也进行了含吡啶基团的吡唑肟醚衍生物研究, 其中部 分叔丁氧羰基吡啶化合物在 $200 \mathrm{mg} \cdot \mathrm{L}^{-1}$ 时, 对朱砂叶螨 的杀死率均达到 $100 \%$, 与商业化的品种唑螨酯同浓度 下活性相当 ${ }^{[12]}$.

*E-mail: xmzou@nankai.edu.cn

Received August 3, 2014; revised September 26, 2014; published online October 13, 2014.

Project supported by the National Natural Science Foundation of China (Nos. 20772067, 21472102)

国家自然科学基金(Nos. 20772067, 21472102)资助项目. 


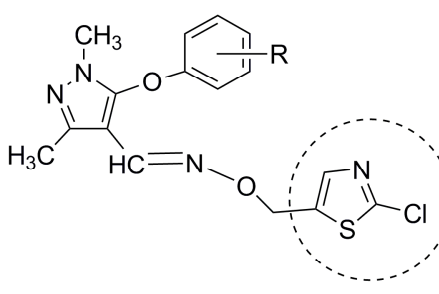

target compounds series A

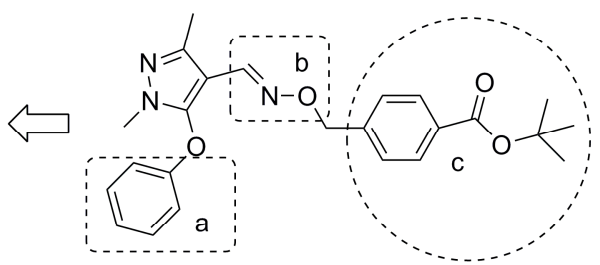

structure of fenpyroximate

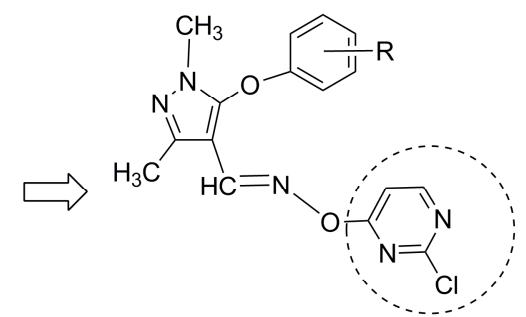

target compounds series B

图 1 唑螨酯和目标化合物系列 A 和 B 的结构

Figure 1 structures of fenpyroximate and target compounds of series A \& B

近年来发现的含有氯代噻唑基团的噻虫嗪(thiamethoxam)和噻虫胺(chlothanidin) 以及 AKD-1022 是继含氯 代吡啶基团的吡虫啉(imdacloprid)之后的第二代新烟碱 类杀虫剂 ${ }^{[13,14]}$, 和吡虫啉一样具有高效、低毒、安全、 广谱等特点, 氯代噻唑是其中的重要药效团 ${ }^{[15]}$ (图 2). 嘧 啶类化合物广泛应用在生物、医药和农药领域, 很多除 草剂、杀虫剂都含有嘧啶环结构, 嘧螨梄(pyrimi- dinfen) 就是含有嘧啶环的高效杀螨剂(图 2). 为了寻找新的活 性化合物, 依据生物等排原理, 以吡唑肜醚为母核, 引 入第二代新烟碱类杀虫剂的重要药效团氯代噻唑和具 有生物高活性的嘧啶环, 设计合称了系列目标化合物 A1 $\sim$ A21 和 B1 $\sim$ B14 (Scheme 1), 采用 ${ }^{1} H$ NMR、IR、 元素分析和 MS 进行了结构确证, 并对它们的杀虫、杀 螨以及杀菌活性进行了测试.

\section{1 结果与讨论}

\section{1 合成}

经结构确证, 所合成的化合物为反式肜醚构型. 制

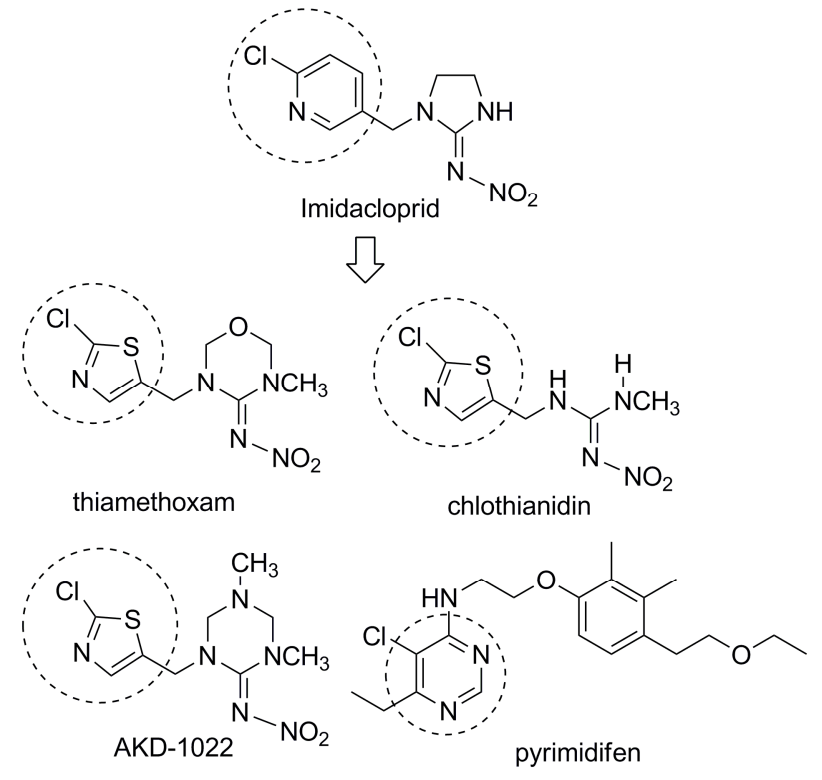

图 2 部分新烟碱类杀虫剂和含嘧啶环的杀螨剂的结构

Figure 2 Structures of some neonicotinoid insecticides and acaricides containing pyrimidine ring 备 1,3-二甲基-4-甲醛基-5-氯吡唑的反应为人名反应，即<smiles>COC(=O)CC(C)=O</smiles><smiles>Cc1nn(C)c(Cl)c1C=O</smiles>

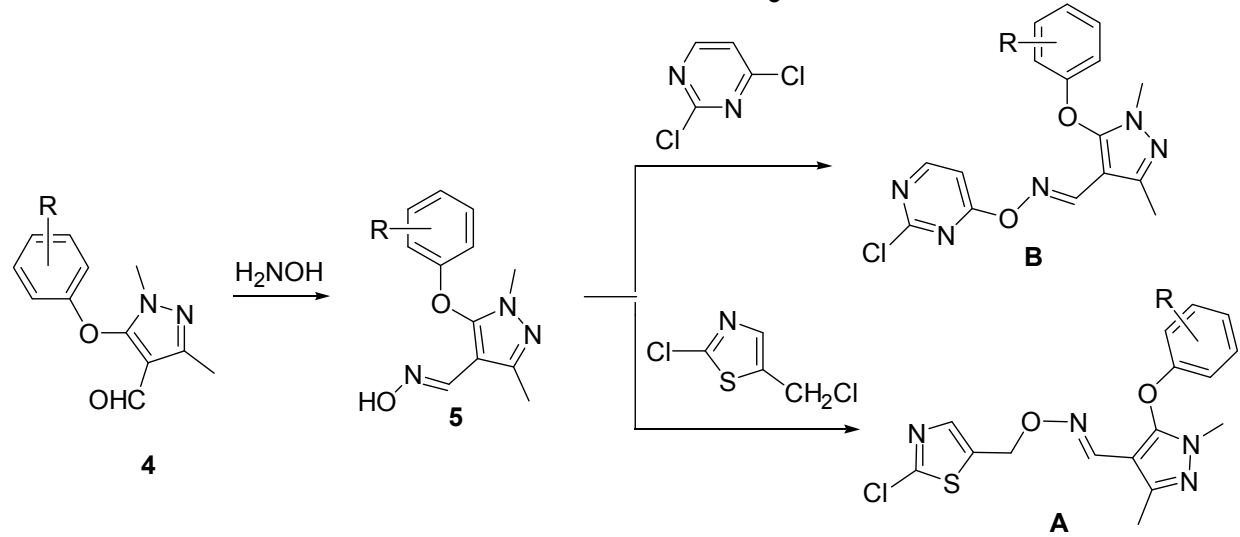

图式 1 目标化合物系列 $\mathbf{A}$ 和 $\mathbf{B}$ 的合成

Scheme 1 Synthesis of target compounds of series A \& B 
Vilsmeier Haack 反应 ${ }^{[16]}$. 文献中制备类似的取代吡唑类 化合物时, $\mathrm{POCl}_{3}$ 和 $\mathrm{DMF}$ 投料量都较大, 分别为反应物 的 7 倍量和 4 倍量. 通过试验发现, 当 $\mathrm{POCl}_{3}$ 和 $\mathrm{DMF}$ 投 料量分别为反应物的 4 倍量和 1 倍量时, 反应收率并没 有降低，由此，对其反应条件进行了一定的优化.

在制备化合物 2 时, 采用不同于一般文献[17]的制 备方法, 用快速回流的石油醚将反应体系中的甲醇、水 混合物带出反应体系, 使得反应进行比较彻底, 基本可 以定量地得到具有较高纯度目标中间体, 无需进行进一 步的纯化即可用于下一步反应.

而合成化合物 4 时，取代苯酚上的取代基对反应进 行及处理影响较大, 通过 $\mathrm{GC}$ 监控反应进程发现, 当取 代基为供电子基时, 反应进行比较彻底, 且水洗 DMF 后萃取比较方便; 当取代基为吸电子基时反应进行缓慢 且不彻底, 甚至反应在相同条件下不进行, 处理时, 水 洗 DMF 后可以用乙酸乙酯洗涤后过滤就可以得到大部 分产品, 溶解性差; 当用邻硝基苯酚进行取代时, 未见 反应进行. 第二步合成肜时, 反应时间很短、产物很容 易析出，抽滤得到纯的目标中间体.

在制备目标化合物 B 系列时, 发现当苯环上是吸电 子基团时, 反应温度要比是供电子基团降低, 否则得不 到目标产物.

\section{2 谱图解析}

目标化合物 $\mathrm{A16}$ 在 2982 和 $2929 \mathrm{~cm}^{-1}$ 出现的分别 是 $\mathrm{CH}_{3}$ 的碳氢和 $\mathrm{CH}$ 的碳氢伸缩振动吸收峰, 在 1164 , 1620 和 $1550 \mathrm{~cm}^{-1}$ 出现的分别是醚键、碳氮双键和杂环 的伸缩振动吸收峰, 在 $1416 \mathrm{~cm}^{-1}$ 出现的是甲基的弯曲 振动吸收峰.

目标化合物 $\mathbf{B} 3 \delta 2.35,2.46$ 以及 3.67 的单峰分别是 苯环上的甲基和吡唑环上的两个甲基; $\delta 6.86,6.88$ 和 $8.35,8.37$ 处的两个双峰为嘧啶环上氢的吸收峰; $\delta$ 6.72 7.23 处的多重峰为苯环上氢的吸收峰; $\delta 8.16$ 是碳 氮双键的氢; 可以发现苯环和嘧啶环易交盖在一起.

在 B6 的 IR 谱图显示苯环上 $\mathrm{H}$ 的伸缩振动出现在 $3071 \mathrm{~cm}^{-1}$ 处, 在 $1555,1424 \mathrm{~cm}^{-1}$ 处分别为苯环、嘧啶 及吡唑环的伸缩振动吸收峰, 在 $1294,1207 \mathrm{~cm}^{-1}$ 处为醚 键伸缩振动吸收峰, 在 $831,717 \mathrm{~cm}^{-1}$ 处为苯环上 $\mathrm{C}-\mathrm{H}$ 的面外弯曲振动吸收.

\section{3 生物活性测试结果}

\subsection{1 杀虫杀螨活性测试结果}

表 1 的生测结果表明，当施药浓度为 $5 \mathrm{mg} \cdot \mathrm{L}^{-1}$ 时， 有近半数 A 系列目标化合物对双翅目的蚊虫活性超过 $100 \%, \mathbf{A 1}$ 和 $\mathbf{A 5}$ 在 $200 \mathrm{mg} \cdot \mathrm{L}^{-1}$ 对红叶螨的活性较好, 其 它化合物都不具备杀螨活性, 其中化合物 A2, A5, A12
和 $\mathbf{A 1 4}$ 在 $200 \mathrm{mg} \cdot \mathrm{L}^{-1}$ 时对属于同翅目的豆蚜活性达到 $100 \%, \mathbf{A 2}, \mathrm{A5}$ 化合物在浓度降低到 $40 \mathrm{mg} \cdot \mathrm{L}^{-1}$ 的时候, 杀蚜虫仍能达到 $81.8 \%$ 和 $83.3 \%$. 这与前期工作用氯代 吡啶取代获得的吡唑肜醚化合物的活性相一致 ${ }^{[12]}$, 说明 原化合物 $\mathrm{c}$ 部分(图 1)具备有杀螨活性的必要条件的基 团. A1 的 a 部分与唑螨酯相同, A5 的 a 部分是对氯苯氧 基，这两个化合物具有杀螨活性，而其它具有不同取代 苯氧基的氯代噻唑化合物则不具备, 说明 $\mathrm{a}$ 部分也含有 杀螨活性的必要条件的活性基团, 而嘧啶环取代 $\mathrm{c}$ 部分 后使得化合物丧失了杀虫杀螨活性.

\subsection{2 杀菌活性测试结果}

表 2 的生测结果表明, 含有嘧啶环的吡唑肜醚类目 标化合物 $\mathbf{B}$ 基本上不具有杀虫活性，但它们均具有一定 的杀菌作用, 大多数目标化合物对苹果轮纹病菌具有相 对较高的活性, 化合物 B2 和 B9 对苹果轮纹病菌的活性 分别达到 $49.2 \%$ 和 $50.0 \%$, 其结构与活性关系不具有明 显的规律性.

\section{2 结论}

以吡唑肜醚为母核，引入氯代噻唑和嘧啶环药效 团，设计合成了系列化合物 $\mathbf{A 1} \sim \mathbf{A 2 1}$ 和 $\mathbf{B 1} \sim \mathbf{B} 14$, 以 ${ }^{1} \mathrm{H}$ NMR、IR、元素分析及 MS 进行结构确证, 并进行了杀 虫、杀螨及杀菌活性测定，其中具有氯代噻唑环的化合 物 A 系列近半数显示了良好的杀蚊虫活性 $(100 \%, 5$ $\left.\mathrm{mg} \cdot \mathrm{L}^{-1}\right), \mathbf{A 1}$ 和 $\mathbf{A 5}$ 具有良好的杀螨活性(100\%, $200 \mathrm{mg} \cdot$ $\left.\mathrm{L}^{-1}\right), \mathbf{A 2}, \mathbf{A 5}, \mathbf{A 1 2}$ 以及 $\mathrm{A14}$ 在 $200 \mathrm{mg} \cdot \mathrm{L}^{-1}$ 时对豆蜴活性 达到 $100 \%$, 当浓度为 $40 \mathrm{mg} \cdot \mathrm{L}^{-1}$ 时, $\mathbf{A 2}, \mathbf{A 5}$ 的杀蚜虫仍 能达到 $81.8 \%$ 和 $83.3 \%$; 氯代噻唑取代化合物与前期工 作进行的氯代吡啶化合物的杀虫杀螨活性相近. 而具有 嘧啶环的化合物 B 系列显示了一定的杀菌活性, 其中 B2 和 B9 对苹果轮纹病菌的活性分别达到 $49.2 \%$ 和 $50.0 \%$.

\section{3 实验部分}

\section{1 仪器与试剂}

Yanaco CORDER MT-3 CHN 自动元素分析仪; BRUKER 300 型核磁共振仪, $\mathrm{CDCl}_{3}$ 为溶剂, TMS 为内 标; Shimadzu-IR435 红外光谱仪( $\mathrm{KBr}$ ); Yanaco P-500 型 熔点仪, 温度计未经校正; 美国 ThermoFinnigan 公司的 LCQ Advantage 型液质联用仪. 实验用的试剂和原料均 为分析纯或化学纯, 使用前均经常规处理.

\section{2 合成}

\subsubsection{1,3-二甲基-5-羟基吡唑(2)的合成}

将 $23.0 \mathrm{~g}(0.5 \mathrm{~mol})$ 甲基肼溶于 $30 \mathrm{~g}$ 无水甲醇, 加入 
表 1 目标化合物 $\mathbf{A 1} \sim \mathbf{A 2 1}$ 的杀虫杀螨活性测试结果

Table 1 Insecticidal and acarcidal results of target compounds $\mathbf{A 1} \sim \mathbf{A 2 1}$

\begin{tabular}{|c|c|c|c|c|}
\hline \multirow[b]{2}{*}{ Compd. } & \multicolumn{4}{|c|}{ Insecticidal and acaricidal activity $/ \%$} \\
\hline & $\begin{array}{l}\text { Culex pipiens pallens } \\
\quad\left(5 \mathrm{mg} \cdot \mathrm{L}^{-1}\right)\end{array}$ & $\begin{array}{l}\text { Mythimna separata Walker } \\
\left(500 \mathrm{mg} \cdot \mathrm{L}^{-1}\right)\end{array}$ & $\begin{array}{l}\text { Aphis laburni Kaltenbach } \\
\left(200 \mathrm{mg} \cdot \mathrm{L}^{-1}\right)\end{array}$ & $\begin{array}{c}\text { Tetranychus cinnabarinus } \\
\left(200 \mathrm{mg} \cdot \mathrm{L}^{-1}\right)\end{array}$ \\
\hline A1 & 100 & 0 & 13 & 100 \\
\hline $\mathbf{A 2}$ & 100 & 0 & 100 & 0 \\
\hline $\mathbf{A 3}$ & 100 & 0 & 92 & 0 \\
\hline A4 & 100 & 0 & 16 & 0 \\
\hline A5 & 100 & 10 & 100 & 95 \\
\hline A6 & 0 & 0 & 60 & 0 \\
\hline A7 & 30 & 0 & 0 & 0 \\
\hline A8 & 0 & 0 & 29 & 0 \\
\hline A9 & 100 & 0 & 65 & 0 \\
\hline A10 & 0 & 0 & 0 & 0 \\
\hline A11 & 0 & 0 & 12 & 0 \\
\hline A12 & 90 & 0 & 92 & 0 \\
\hline A13 & 100 & 0 & 0 & 0 \\
\hline A14 & 100 & 0 & 100 & 0 \\
\hline A15 & 0 & 0 & 0 & 0 \\
\hline A16 & 0 & 0 & 0 & 0 \\
\hline A17 & 100 & 0 & 51.3 & 0 \\
\hline A18 & 0 & 0 & 0 & 0 \\
\hline A19 & 0 & 0 & 0 & 0 \\
\hline A20 & 0 & 0 & 0 & 0 \\
\hline A21 & 0 & 0 & 0 & 0 \\
\hline Fenpyroximate & - & - & - & 100 \\
\hline
\end{tabular}

表 2 目标化合物 B1 $\sim$ B14 的杀菌活性测定结果

Table 2 The fungicidal activity of compounds B1 $\sim$ B14

\begin{tabular}{|c|c|c|c|c|}
\hline \multirow[b]{2}{*}{ Compd. } & \multicolumn{4}{|c|}{ Insecticidal and acaricidal activity/\% } \\
\hline & $\begin{array}{l}\text { Culex pipiens pallens } \\
\qquad\left(5 \mathrm{mg} \cdot \mathrm{L}^{-1}\right)\end{array}$ & $\begin{array}{l}\text { Mythimna separata Walker } \\
\left(500 \mathrm{mg} \cdot \mathrm{L}^{-1}\right)\end{array}$ & $\begin{array}{l}\text { Aphis laburni Kaltenbach } \\
\left(200 \mathrm{mg} \cdot \mathrm{L}^{-1}\right)\end{array}$ & $\begin{array}{l}\text { Tetranychus cinnabarinus } \\
\left(200 \mathrm{mg} \cdot \mathrm{L}^{-1}\right)\end{array}$ \\
\hline A1 & 100 & 0 & 13 & 100 \\
\hline $\mathbf{A 2}$ & 100 & 0 & 100 & 0 \\
\hline $\mathbf{A 3}$ & 100 & 0 & 92 & 0 \\
\hline A4 & 100 & 0 & 16 & 0 \\
\hline A5 & 100 & 10 & 100 & 95 \\
\hline A6 & 0 & 0 & 60 & 0 \\
\hline A7 & 30 & 0 & 0 & 0 \\
\hline $\mathbf{A 8}$ & 0 & 0 & 29 & 0 \\
\hline A9 & 100 & 0 & 65 & 0 \\
\hline A10 & 0 & 0 & 0 & 0 \\
\hline A11 & 0 & 0 & 12 & 0 \\
\hline A12 & 90 & 0 & 92 & 0 \\
\hline A13 & 100 & 0 & 0 & 0 \\
\hline A14 & 100 & 0 & 100 & 0 \\
\hline A15 & 0 & 0 & 0 & 0 \\
\hline A16 & 0 & 0 & 0 & 0 \\
\hline A17 & 100 & 0 & 51.3 & 0 \\
\hline A18 & 0 & 0 & 0 & 0 \\
\hline A19 & 0 & 0 & 0 & 0 \\
\hline $\mathbf{A 2 0}$ & 0 & 0 & 0 & 0 \\
\hline A21 & 0 & 0 & 0 & 0 \\
\hline Fenpyroximate & - & - & - & 100 \\
\hline
\end{tabular}


$500 \mathrm{~mL}$ 四口瓶中, 并加入 $250 \mathrm{~mL} 60 \sim 90{ }^{\circ} \mathrm{C}$ 石油醚, 机 械搅拌下滴加 $58 \mathrm{~g}(0.5 \mathrm{~mol})$ 乙酰乙酸甲酯, 由于反应放 热, 注意控制滴加速度. 滴加完毕, 装上分水器, 加热 回流, 将甲醇、水混合物分出, 至不再有甲醇、水分出, 反应结束. 冷却, 抽滤, 得白色固体粉末 55.6 g. m.p. $114 \sim 116{ }^{\circ} \mathrm{C}$, 粗收率 99\%(文献值 ${ }^{[17]}$ : m.p. 116 $118{ }^{\circ} \mathrm{C}$, 收率 79.9\%).

\subsubsection{1,3-二甲基-4-甲醛基-5-氯吡唑(3)的合成}

按文献[17]方法合成, m.p. $74 \sim 76{ }^{\circ} \mathrm{C}$, 收率 $66.6 \%$ (文献值 ${ }^{[17]}$ : m.p. $77 \sim 79{ }^{\circ} \mathrm{C}$, 收率 $86 \%$ ).

\subsubsection{1,3-二甲基-5-苯氧基-1 $H$-吡唑-4-甲醛(4)的合成}

在装有机械摚拌及分水器的 $100 \mathrm{~mL}$ 四口瓶中加入 $0.94 \mathrm{~g}(10 \mathrm{mmol})$ 苯酚以及 $10 \mathrm{~mL}$ 甲醇, 再加入用少量甲 醇溶解的 $0.4 \mathrm{~g}(10 \mathrm{mmol})$ 氢氧化钠. 最后加入 $40 \mathrm{~mL}$ 左 右沸点在 $60 \sim 90{ }^{\circ} \mathrm{C}$ 的石油醚, 加热回流, 分水器分水, 直到没有水分出, 蒸出石油梄, 加入 $40 \mathrm{~mL}$ DMF、 1.585 $\mathrm{g}(10 \mathrm{mmol}) 5$-氯-1,3-二甲基- $1 H$-吡唑-4-甲酫. 控制温度 $80 \sim 90{ }^{\circ} \mathrm{C}$ 反应, TLC 跟踪反应, 至反应结束后. 蒸出溶 剂, 萃取、水洗、干燥、浓缩得白色固体 2.03 g. m.p. $110 \sim 111{ }^{\circ} \mathrm{C}$, 产率 $94 \%$ (文献值 ${ }^{[17]}$ : m.p. $110 \sim 112{ }^{\circ} \mathrm{C}$, 收率 $96 \%$ ).

3.2.4 1,3-二甲基-5-苯氧基- $1 H$-吡唑-4-甲醛基肜 (5) 的合成

按文献[17]方法合成, m.p. $133 \sim 134{ }^{\circ} \mathrm{C}$, 收率 $96.6 \%$ (文献值 ${ }^{[17]}$ : m.p. $133 \sim 134{ }^{\circ} \mathrm{C}$, 收率 96\%).

3.2.5 (E)-1,3-二甲基-5-芳氧基- $1 H$-吡唑-4-甲醛 $O$ - (2氯噻唑-5-甲基)肟(A1 A A21)的合成

在装有温度计的 $100 \mathrm{~mL}$ 四口瓶中, 加入 $0.693 \mathrm{~g} \mathrm{(3}$ mmol） 5 -苯氧基-1,3-二甲基- $1 H$-吡唑-4-甲醛基肜以及 $0.828 \mathrm{~g}(6 \mathrm{mmol})$ 碳酸钾, 最后加入 $0.604 \mathrm{~g}(3.6 \mathrm{mmol}) 2-$ 氯-5-氯甲基噻坐以及 $40 \mathrm{~mL}$ 乙腈作为溶剂. 加热回流. TLC 跟踪反应至反应完成. 过滤、浓缩, 以丙酮/石油醚 $\left(60 \sim 90{ }^{\circ} \mathrm{C}\right)(V / V=1 / 5)$ 为洗脱剂进行柱层析得白色固 体, 合成了化合物 $\mathbf{A 1}$. 同法合成了化合物 $\mathbf{A 2} \sim \mathbf{A 2 1}$. 化 合物 $\mathbf{A 1} \sim \mathbf{A 2 1}$ 的物理常数和元素分析数值及核磁共振 氢谱数据如下:

(E)-1,3-二甲基-5-苯氧基- $1 H$-吡唑-4-甲醛 $O$-(2-氯噻 唑-5-甲基)肜(A1)：白色固体, 产率 83.5\%. m.p. 64 $66{ }^{\circ} \mathrm{C} ;{ }^{1} \mathrm{H}$ NMR $\left(\mathrm{CDCl}_{3}, 300 \mathrm{MHz}\right) \delta: 7.77(\mathrm{~s}, 1 \mathrm{H}, \mathrm{CH}=$ $\mathrm{N}), 7.38(\mathrm{~s}, 1 \mathrm{H}$, Thiazole-H), 6.90 7.35 (m, 5H, ArH), $5.03\left(\mathrm{~s}, 2 \mathrm{H}, \mathrm{CH}_{2}\right), 3.60\left(\mathrm{~s}, 3 \mathrm{H}\right.$, Pyrazole $\left.(\mathrm{N})-\mathrm{CH}_{3}\right), 2.40(\mathrm{~s}$, $3 \mathrm{H}$, Pyrazole- $\mathrm{CH}_{3}$ ); MS (ESI) $m / z$ : $363.6[\mathrm{M}+\mathrm{H}]^{+}$. Anal. calcd for $\mathrm{C}_{16} \mathrm{H}_{15} \mathrm{ClN}_{4} \mathrm{O}_{2} \mathrm{~S}$ : C 52.96, H 4.17, N 15.44; found C 53.35, H 4.50, N 15.00 .
(E)-1,3-二甲基-5-(4-甲基苯氧基)- $1 H$-吡唑-4-甲醛 $O$-(2-氯噻唑-5-甲基)肜 (A2): 无色粘稠液体，产率 53.3\%. ${ }^{1} \mathrm{H} \mathrm{NMR}\left(\mathrm{CDCl}_{3}, 300 \mathrm{MHz}\right) \delta: 7.76(\mathrm{~s}, 1 \mathrm{H}, \mathrm{CH}=$ N), $7.39(\mathrm{~s}, 1 \mathrm{H}$, Thiazole-H), 6.76 7.22 (m, 4H, ArH), $5.05\left(\mathrm{~s}, 2 \mathrm{H}, \mathrm{CH}_{2}\right), 3.59\left(\mathrm{~s}, 3 \mathrm{H}\right.$, Pyrazole $\left.(\mathrm{N})-\mathrm{CH}_{3}\right), 2.39(\mathrm{~s}$, $3 \mathrm{H}$, Pyrazole- $\mathrm{CH}_{3}$ ), 2.32 (s, $3 \mathrm{H}, \mathrm{ArCH}_{3}$ ). Anal. calcd for $\mathrm{C}_{17} \mathrm{H}_{17} \mathrm{ClN}_{4} \mathrm{O}_{2} \mathrm{~S}$ : C 54.18, H 4.55, N 14.87; found C 54.04, $\mathrm{H} 4.51, \mathrm{~N} 14.85$.

(E)-1,3-二甲基-5-(3-甲基苯氧基)-1 $H$-吡唑-4-甲醛 $O$-(2-氯塞唑-5-甲基) 肜 (A3): 无色粘稠液体, 产率 51.1\%. ${ }^{1} \mathrm{H}$ NMR $\left(\mathrm{CDCl}_{3}, 300 \mathrm{MHz}\right) \delta: 7.77(\mathrm{~s}, 1 \mathrm{H}, \mathrm{CH}=$ N), $7.39(\mathrm{~s}, 1 \mathrm{H}$, Thiazole-H), 6.69 7.22 (m, 4H, ArH), $5.05\left(\mathrm{~s}, 2 \mathrm{H}, \mathrm{CH}_{2}\right), 3.59$ (s, 3H, Pyrazole (N)- $\left.\mathrm{CH}_{3}\right), 2.40$ (s, $3 \mathrm{H}$, Pyrazole- $\mathrm{CH}_{3}$ ), 2.32 (s, $3 \mathrm{H}, \mathrm{ArCH}_{3}$ ). Anal. calcd for $\mathrm{C}_{17} \mathrm{H}_{17} \mathrm{ClN}_{4} \mathrm{O}_{2} \mathrm{~S}$ : C 54.18, H 4.55, N 14.87; found C 54.36, $\mathrm{H} 4.69, \mathrm{~N} 15.05$.

(E)-1,3-二甲基-5-(2-甲基苯氧基)-1 $H$-吡唑-4-甲醛 $O$-(2-氯噻唑-5-甲基)肜(A4): 白色固体, 产率 $86.0 \%$. m.p. $57 \sim 59{ }^{\circ} \mathrm{C} ;{ }^{1} \mathrm{H}$ NMR $\left(\mathrm{CDCl}_{3}, 300 \mathrm{MHz}\right) \delta$ : 7.70 (s, $1 \mathrm{H}, \mathrm{CH}=\mathrm{N}), 7.38(\mathrm{~s}, 1 \mathrm{H}$, Thiazole-H), $7.02 \sim 7.25(\mathrm{~m}, 4 \mathrm{H}$, $\mathrm{ArH}), 5.03$ (s, $2 \mathrm{H}, \mathrm{CH}_{2}$ ), 3.60 (s, $3 \mathrm{H}$, Pyrazole (N)- $\mathrm{CH}_{3}$ ), 2.39 (s, $3 \mathrm{H}$, Pyrazole- $\left.\mathrm{CH}_{3}\right), 2.38$ (s, $3 \mathrm{H}, \mathrm{ArCH}_{3}$ ). Anal. calcd for $\mathrm{C}_{17} \mathrm{H}_{17} \mathrm{ClN}_{4} \mathrm{O}_{2} \mathrm{~S}$ : C 54.18, H 4.55, N 18.87; found C 54.37, H 4.66, N 14.82 .

(E)-1,3-二甲基-5-(2-氯苯氧基)- $1 H$-吡唑-4-甲醛 $O$ (2-氯噻唑-5-甲基)肜(A5)：淡黄色固体，产率 71.1\%. m.p. $61 \sim 62{ }^{\circ} \mathrm{C} ;{ }^{1} \mathrm{H}$ NMR $\left(\mathrm{CDCl}_{3}, 300 \mathrm{MHz}\right) \delta: 7.77$ (s, $1 \mathrm{H}, \mathrm{CH}=\mathrm{N}), 7.40(\mathrm{~s}, 1 \mathrm{H}$, Thiazole-H), $6.82 \sim 7.30(\mathrm{~m}, 4 \mathrm{H}$, $\mathrm{ArH}), 5.03$ (s, $2 \mathrm{H}, \mathrm{CH}_{2}$ ), 3.60 (s, 3H, Pyrazole (N)- $\mathrm{CH}_{3}$ ), 2.38 (s, 3H, Pyrazole- $\mathrm{CH}_{3}$ ). Anal. calcd for $\mathrm{C}_{16} \mathrm{H}_{14} \mathrm{Cl}_{2}-$ $\mathrm{N}_{4} \mathrm{O}_{2} \mathrm{~S}$ : C 48.37, H 3.55, N 14.10; found C 48.19, H 3.65, $\mathrm{N} 14.24$.

(E)-1,3-二甲基-5-(3-溴基苯氧基)-1 $H$-吡唑-4-甲醛 $O$-(2-氯噻唑-5-甲基)肜 (A6)：无色粘稠液体，产率 86.1\%. ${ }^{1} \mathrm{H}$ NMR $\left(\mathrm{CDCl}_{3}, 300 \mathrm{MHz}\right) \delta: 7.78(\mathrm{~s}, 1 \mathrm{H}, \mathrm{CH}=$ N), $7.38(\mathrm{~s}, 1 \mathrm{H}$, Thiazole-H), $6.81 \sim 7.19(\mathrm{~m}, 4 \mathrm{H}, \mathrm{ArH})$, $5.03\left(\mathrm{~s}, 2 \mathrm{H}, \mathrm{CH}_{2}\right), 3.61\left(\mathrm{~s}, 3 \mathrm{H}\right.$, Pyrazole $\left.(\mathrm{N})-\mathrm{CH}_{3}\right), 2.39$ (s, $3 \mathrm{H}$, Pyrazole- $\mathrm{CH}_{3}$ ). Anal. calcd for $\mathrm{C}_{16} \mathrm{H}_{14} \mathrm{BrClN}_{4} \mathrm{O}_{2} \mathrm{~S}$ : C 43.50, H 3.19, N 12.68; found C 43.59, H 3.29, N 12.50 .

(E)-1,3-二甲基-5-(3,5-二甲基苯氧基)- $1 \mathrm{H}$-吡唑-4-甲 醛 $O$-(2-氯噻唑-5-甲基)肜 (A7): 白色固体, 产率 77.4\%. m.p. $84 \sim 86{ }^{\circ} \mathrm{C} ;{ }^{1} \mathrm{H}$ NMR $\left(\mathrm{CDCl}_{3}, 300 \mathrm{MHz}\right) \delta$ : 7.82 (s, $1 \mathrm{H}, \mathrm{CH}=\mathrm{N}), 7.39(\mathrm{~s}, 1 \mathrm{H}$, Thiazole-H), $6.79(\mathrm{~s}, 1 \mathrm{H}, \mathrm{ArH})$, 6.48 (s, 2H, ArH), 5.04 (s, 2H, $\mathrm{CH}_{2}$ ), 3.62 (s, 3H, Pyrazole $\left.(\mathrm{N})-\mathrm{CH}_{3}\right), 2.44\left(\mathrm{~s}, 3 \mathrm{H}\right.$, Pyrazole- $\left.\mathrm{CH}_{3}\right), 2.27(\mathrm{~s}, 6 \mathrm{H}$, 
$\mathrm{ArCH}_{3}$ ); MS (ESI) $m / z: 391.5[\mathrm{M}+\mathrm{H}]^{+}$. Anal. calcd for $\mathrm{C}_{18} \mathrm{H}_{19} \mathrm{ClN}_{4} \mathrm{O}_{2} \mathrm{~S}$ : C 55.31, H 4.90, N 14.33; found C 55.49, $\mathrm{H} 4.80, \mathrm{~N} 14.30$.

(E)-1,3-二甲基-5-(4-甲氧羰基苯氧基)- $1 H$-吡唑-4甲醛 $O$-(2-氯噻唑-5-甲基)肜 (A8): 淡黄色液体, 产率 55.6\%. ${ }^{1} \mathrm{H} \mathrm{NMR}\left(\mathrm{CDCl}_{3}, 300 \mathrm{MHz}\right) \delta: 8.04(\mathrm{~s}, 1 \mathrm{H}, \mathrm{CH}=$ $\mathrm{N}), 8.01(\mathrm{~s}, 1 \mathrm{H}$, Thiazole-H), $6.92 \sim 7.78(\mathrm{~m}, 4 \mathrm{H}, \operatorname{ArH})$, 5.00 (s, 2H, $\mathrm{CH}_{2}$ ), 3.91 (s, $3 \mathrm{H}, \mathrm{CO}_{2} \mathrm{CH}_{3}$ ), 3.61 (s, 3H, Pyrazole $\left.(\mathrm{N})-\mathrm{CH}_{3}\right), 2.39$ (s, 3H, Pyrazole- $\left.\mathrm{CH}_{3}\right)$. Anal. calcd for $\mathrm{C}_{18} \mathrm{H}_{17} \mathrm{ClN}_{4} \mathrm{O}_{4} \mathrm{~S}$ : C 51.37, $\mathrm{H} \mathrm{4.07,} \mathrm{N} \mathrm{13.31;} \mathrm{found} \mathrm{C}$ 51.51, H 4.28, N 13.12 .

(E)-1,3-二甲基-5-(2,4-二甲基苯氧基)- $1 H$-吡唑-4-甲 醛 $O$-(2-氯噻唑-5-甲基)肜 (A9): 淡黄色固体, 产率 94.1\%. m.p. 58 59 ${ }^{\circ} \mathrm{C} ;{ }^{1} \mathrm{H}$ NMR $\left(\mathrm{CDCl}_{3}, 300 \mathrm{MHz}\right) \delta$ : $7.69(\mathrm{~s}, 1 \mathrm{H}, \mathrm{CH}=\mathrm{N}), 7.39(\mathrm{~s}, 1 \mathrm{H}$, Thiazole-H), $6.40 \sim 7.04$ (m, 3H, ArH), 5.04 (s, 2H, $\left.\mathrm{CH}_{2}\right), 3.59$ (s, 3H, Pyrazole $\left.(\mathrm{N})-\mathrm{CH}_{3}\right), 2.39\left(\mathrm{~s}, 3 \mathrm{H}\right.$, Pyrazole- $\left.\mathrm{CH}_{3}\right), 2.34(\mathrm{~s}, 3 \mathrm{H}$, $\mathrm{ArCH}_{3}$ ), 2.28 (s, $3 \mathrm{H}, \mathrm{ArCH}_{3}$ ). Anal. calcd for $\mathrm{C}_{18} \mathrm{H}_{19} \mathrm{Cl}-$ $\mathrm{N}_{4} \mathrm{O}_{2} \mathrm{~S}$ : C 55.31, H 4.90, N 14.33; found C 55.32, H 5.05, $\mathrm{N} 14.26$.

(E)-1,3-二甲基-5-(3-硝基苯氧基)-1 $H$-吡唑-4-甲醛 $O$-(2-氯噻唑-5-甲基)肜(A10): 淡黄色固体, 产率 65.0\%. m.p. $91 \sim 92{ }^{\circ} \mathrm{C} ;{ }^{1} \mathrm{H}$ NMR $\left(\mathrm{CDCl}_{3}, 300 \mathrm{MHz}\right) \delta$ : 7.99 $8.03(\mathrm{~m}, 1 \mathrm{H}, \mathrm{CH}=\mathrm{N}), 7.80(\mathrm{~s}, 1 \mathrm{H}$, Thiazole-H), 7.32 7.74 (m, 4H, ArH), 4.96 (s, 2H, $\mathrm{CH}_{2}$ ), 3.65 (s, 3H, Pyrazole (N)- $\mathrm{CH}_{3}$ ), 2.39 (s, 3H, Pyrazole- $\mathrm{CH}_{3}$ ); MS (ESI) $\mathrm{m} / \mathrm{z}$ : $408.5[\mathrm{M}+\mathrm{H}]^{+}$. Anal. calcd for $\mathrm{C}_{16} \mathrm{H}_{14} \mathrm{ClN}_{5} \mathrm{O}_{4} \mathrm{~S}: \mathrm{C} 47.12$, H 3.46, N 17.17; found C 46.93, H 3.29, N 17.29.

(E)-1,3-二甲基-5-(2-甲氧基苯氧基)- $1 H$-吡唑-4-甲 醛 $O$-(2-氯噻唑-5-甲基)肜 (A11): 淡黄色固体, 产率 83.1\%. m.p. 71 $72{ }^{\circ} \mathrm{C} ;{ }^{1} \mathrm{H}$ NMR $\left(\mathrm{CDCl}_{3}, 300 \mathrm{MHz}\right) \delta$ : $7.73(\mathrm{~s}, 1 \mathrm{H}, \mathrm{CH}=\mathrm{N}), 7.39(\mathrm{~s}, 1 \mathrm{H}$, Thiazole-H), 6.67 7.10 (m, 4H, ArH), 5.03 (s, 2H, CH $), 3.91$ (s, 3H, Pyrazole $\left.(\mathrm{N})-\mathrm{CH}_{3}\right), 3.63\left(\mathrm{~s}, 3 \mathrm{H}, \mathrm{OCH}_{3}\right), 2.38$ (s, 3H, Pyrazole- $\left.\mathrm{CH}_{3}\right)$. Anal. calcd for $\mathrm{C}_{17} \mathrm{H}_{17} \mathrm{ClN}_{4} \mathrm{O}_{3} \mathrm{~S}$ : C 51.97, H 4.36, N 14.26; found $\mathrm{C} 52.08, \mathrm{H} 4.32, \mathrm{~N} 14.23$.

(E)-1,3-二甲基-5-(3-三氟甲基苯氧基)-1 $H$-吡唑-4甲醛 $O$-(2-氯噻唑-5-甲基)肜(A12): 淡黄色粘稠液体, 产 率 69.7\%. ${ }^{1} \mathrm{H}$ NMR $\left(\mathrm{CDCl}_{3}, 300 \mathrm{MHz}\right) \delta: 7.78(\mathrm{~s}, 1 \mathrm{H}$, $\mathrm{CH}=\mathrm{N}), 7.35(\mathrm{~s}, 1 \mathrm{H}$, Thiazole-H), $7.02 \sim 7.48(\mathrm{~m}, 4 \mathrm{H}$, ArH), 4.99 (s, 2H, $\mathrm{CH}_{2}$ ), 3.63 (s, 3H, Pyrazole (N)- $\mathrm{CH}_{3}$ ), 2.39 (s, 3H, Pyrazole- $\mathrm{CH}_{3}$ ). Anal. calcd for $\mathrm{C}_{17} \mathrm{H}_{17} \mathrm{Cl}-$ $\mathrm{N}_{4} \mathrm{O}_{3} \mathrm{~S}$ : C 47.39, H 3.28, N 13.00; found C 47.32, H 3.42, $\mathrm{N} 13.10$.

(E)-1,3-二甲基-5-(2,4 二氯苯氧基)-1H-吡唑-4-甲醛
$O$-(2-氯噻唑-5-甲基)肜(A13): 粉红色固体, 产率 81.0\%. m.p. $79 \sim 80{ }^{\circ} \mathrm{C} ;{ }^{1} \mathrm{H}$ NMR $\left(\mathrm{CDCl}_{3}, 300 \mathrm{MHz}\right) \delta: 7.76$ (s, $1 \mathrm{H}, \mathrm{CH}=\mathrm{N}), 7.48(\mathrm{~s}, 1 \mathrm{H}$, Thiazole-H), $7.47 \sim 6.62(\mathrm{~m}, 3 \mathrm{H}$, ArH), $5.02\left(\mathrm{~s}, 2 \mathrm{H}, \mathrm{CH}_{2}\right), 3.63\left(\mathrm{~s}, 3 \mathrm{H}\right.$, Pyrazole $\left.(\mathrm{N})-\mathrm{CH}_{3}\right)$, 2.37 (s, 3H, Pyrazole- $\mathrm{CH}_{3}$ ). Anal. calcd for $\mathrm{C}_{16} \mathrm{H}_{13} \mathrm{Cl}_{3}$ $\mathrm{N}_{4} \mathrm{O}_{2} \mathrm{~S}$ : C 44.51, H 3.04, N 12.98; found C 45.01, H 3.09, $\mathrm{N} 13.08$.

(E)-1,3-二甲基-5-(3-氯苯氧基)- $1 H$-吡唑-4-甲醛 $O$ (2-氯噻唑-5-甲基)肜(A14): 无色粘稠液体，产率 52.7\%. ${ }^{1} \mathrm{H}$ NMR $\left(\mathrm{CDCl}_{3}, 300 \mathrm{MHz}\right) \delta: 7.78(\mathrm{~s}, 1 \mathrm{H}, \mathrm{CH}=\mathrm{N}), 7.38$ (s, 1H, Thiazole-H), $6.77 \sim 7.25(\mathrm{~m}, 4 \mathrm{H}, \mathrm{ArH}), 5.03(\mathrm{~s}, 2 \mathrm{H}$, $\mathrm{CH}_{2}$ ), 3.61 (s, 3H, Pyrazole(N)- $\mathrm{CH}_{3}$ ), 2.39 (s, 3H, Pyrazole- $\mathrm{CH}_{3}$ ). Anal. calcd for $\mathrm{C}_{16} \mathrm{H}_{14} \mathrm{Cl}_{2} \mathrm{~N}_{4} \mathrm{O}_{2} \mathrm{~S}$ : C $48.37, \mathrm{H}$ 3.55, N 14.10; found C 48.35, H 3.81, N 14.15.

(E)-1,3-二甲基-5-叔丁氧基- $1 H$-吡唑-4-甲醛 $O$-(2-氯 噻唑-5-甲基)肜(A15)：黄色固体, 产率 47.1\%. m.p. 53 $55{ }^{\circ} \mathrm{C} ;{ }^{1} \mathrm{H}$ NMR $\left(\mathrm{CDCl}_{3}, 300 \mathrm{MHz}\right) \delta: 7.93(\mathrm{~s}, 1 \mathrm{H}, \mathrm{CH}=$ N), $7.49\left(\mathrm{~s}, 1 \mathrm{H}\right.$, Thiazole-H), $5.16\left(\mathrm{~s}, 2 \mathrm{H}, \mathrm{CH}_{2}\right), 3.62(\mathrm{~s}$, $3 \mathrm{H}$, Pyrazole (N)- $\left.\mathrm{CH}_{3}\right), 2.38$ (s, 3H, Pyrazole- $\left.\mathrm{CH}_{3}\right), 1.37$ (s, $\left.9 \mathrm{H}, \mathrm{C}\left(\mathrm{CH}_{3}\right)_{3}\right)$. Anal. calcd for $\mathrm{C}_{14} \mathrm{H}_{19} \mathrm{ClN}_{4} \mathrm{O}_{2} \mathrm{~S}$ : C 49.05, H 5.59, N 16.06; found C 49.26, H 5.58, N 16.06.

(E)-1,3-二甲基-5-异丙氧基- $1 H$-吡唑-4-甲醛 $O$-(2-氯 噻唑-5-甲基)肜(A16)：白色固体，产率 65.2\%. m.p. 51 $52{ }^{\circ} \mathrm{C} ;{ }^{1} \mathrm{H}$ NMR $\left(\mathrm{CDCl}_{3}, 300 \mathrm{MHz}\right) \delta: 7.95(\mathrm{~s}, 1 \mathrm{H}, \mathrm{CH}=$ N), 7.49 (s, $1 \mathrm{H}$, Thiazole-H), $5.17\left(\mathrm{~s}, 2 \mathrm{H}, \mathrm{CH}_{2}\right), 4.51 \sim 4.60$ $(\mathrm{m}, 1 \mathrm{H}, \mathrm{CH}), 3.61\left(\mathrm{~s}, 3 \mathrm{H}\right.$, Pyrazole $\left.(\mathrm{N})-\mathrm{CH}_{3}\right), 2.30(\mathrm{~s}, 3 \mathrm{H}$, Pyrazole- $\left.\mathrm{CH}_{3}\right), 1.30 \sim 1.32\left(\mathrm{~d}, 6 \mathrm{H}, \mathrm{C}\left(\mathrm{CH}_{3}\right)_{2}\right)$. Anal. calcd for $\mathrm{C}_{13} \mathrm{H}_{17} \mathrm{ClN}_{4} \mathrm{O}_{2} \mathrm{~S}$ : C 47.49, $\mathrm{H}$ 5.21, N 17.04; found $\mathrm{C}$ 47.28, H 5.19, N 16.88 .

(E)-1,3-二甲基-5- $\beta$-䒺氧基- $1 H$-吡唑-4-甲醛 $O$-(2-氯 噻唑-5-甲基)肜(A17): 淡黄色固体, 产率 74.6\%. m.p. $63 \sim 64{ }^{\circ} \mathrm{C} ;{ }^{1} \mathrm{H}$ NMR $\left(\mathrm{CDCl}_{3}, 300 \mathrm{MHz}\right) \delta: 7.85(\mathrm{~s}, 1 \mathrm{H}$, $\mathrm{CH}=\mathrm{N}), 7.83(\mathrm{~s}, 1 \mathrm{H}$, Thiazole-H), $7.25 \sim 7.80(\mathrm{~m}, 7 \mathrm{H}$, Naphthalene-H), 4.98 (s, 2H, $\mathrm{CH}_{2}$ ), 3.63 (s, 3H, Pyrazole (N)- $\left.\mathrm{CH}_{3}\right), 2.43$ (s, 3H, Pyrazole- $\mathrm{CH}_{3}$ ).

(E)-1,3-二甲基-5-(4-氟苯氧基)- $1 H$-吡唑-4-甲醛 $O$ (2-氯噻唑-5-甲基)肜 (A18): 淡黄色粘稠液体, 产率 83.1\%. ${ }^{1} \mathrm{H} \mathrm{NMR}\left(\mathrm{CDCl}_{3}, 300 \mathrm{MHz}\right) \delta: 7.76(\mathrm{~s}, 1 \mathrm{H}, \mathrm{CH}=$ N), $7.39(\mathrm{~s}, 1 \mathrm{H}$, Thiazole-H), 6.83 7.04 (m, 4H, ArH), $5.03\left(\mathrm{~s}, 2 \mathrm{H}, \mathrm{CH}_{2}\right), 3.61\left(\mathrm{~s}, 3 \mathrm{H}\right.$, Pyrazole $\left.(\mathrm{N})-\mathrm{CH}_{3}\right), 2.38$ (s, $3 \mathrm{H}$, Pyrazole- $\mathrm{CH}_{3}$ ). Anal. calcd for $\mathrm{C}_{16} \mathrm{H}_{14} \mathrm{ClFN}_{4} \mathrm{O}_{2} \mathrm{~S}$ : C 50.46, H 4.10, N 14.71; found C 49.91, H 3.95, N 14.38.

(E)-1,3-二甲基-5- $\alpha$-䒺氧基- $1 H$-吡唑-4-甲醛 $O$-(2-氯 噻唑-5-甲基)肜(A19): 淡黄色固体，产率 39.6\%. m.p. $118 \sim 119{ }^{\circ} \mathrm{C} ; 1 \mathrm{H} \mathrm{NMR}\left(\mathrm{CDCl}_{3}, 300 \mathrm{MHz}\right) \delta: 6.60 \sim 8.39$ 
(m, 9H, CH $=\mathrm{N} \&$ Thiazole-H \& Naphthalene- H), 4.90 (s, $\left.2 \mathrm{H}, \mathrm{CH}_{2}\right), 3.66\left(\mathrm{~s}, 3 \mathrm{H}\right.$, Pyrazole $\left.(\mathrm{N})-\mathrm{CH}_{3}\right), 2.44(\mathrm{~s}, 3 \mathrm{H}$, Pyrazole- $\mathrm{CH}_{3}$ ). Anal. calcd for $\mathrm{C}_{20} \mathrm{H}_{17} \mathrm{ClN}_{4} \mathrm{O}_{2} \mathrm{~S}$ : C $58.18, \mathrm{H}$ 4.15, N 13.57; found C 58.06, H 4.00, N 13.26.

(E)-1,3-二甲基-5-烯丙氧基-1 $H$-吡唑-4-甲醛 $O$-(2-氯 噻唑-5-甲基)肜(A20): 淡黄色液, 产率 68.5\%。 ${ }^{1} \mathrm{H}$ NMR $\left(\mathrm{CDCl}_{3}, 300 \mathrm{MHz}\right) \delta: 7.47(\mathrm{~s}, 1 \mathrm{H}, \mathrm{CH}=\mathrm{N}), 7.29(\mathrm{~s}, 1 \mathrm{H}$, Thiazole-H), $5.13\left(\mathrm{~s}, 2 \mathrm{H}, \mathrm{CH}_{2}\right), 5.17 \sim 5.52\left(\mathrm{~m}, 3 \mathrm{H}, \mathrm{CH}_{2}=\right.$ $\mathrm{CH}$ ), 3.28 (s, 3H, Pyrazole(N)- $\mathrm{CH}_{3}$ ), 2.08 (s, 3H, Pyrazole$\mathrm{CH}_{3}$ ). Anal. calcd for $\mathrm{C}_{13} \mathrm{H}_{15} \mathrm{ClN}_{4} \mathrm{O}_{2} \mathrm{~S}$ : C 47.78, $\mathrm{H}$ 4.63, N 17.14; found C 48.08, H 4.47, N 16.90.

(E)-1,3-二甲基-5-氯- $1 H$-吡唑-4-甲醛 $O$-(2-氯噻唑5-甲基)肜(A21)：无色固体，产率 55.2\%. m.p. 63 $64{ }^{\circ} \mathrm{C} ;{ }^{1} \mathrm{H}$ NMR $\left(\mathrm{CDCl}_{3}, 300 \mathrm{MHz}\right) \delta: 7.98(\mathrm{~s}, 1 \mathrm{H}, \mathrm{CH}=$ $\mathrm{N}), 7.51\left(\mathrm{~s}, 1 \mathrm{H}\right.$, Thiazole-H), $5.19\left(\mathrm{~s}, 2 \mathrm{H}, \mathrm{CH}_{2}\right), 3.79(\mathrm{~s}$, $3 \mathrm{H}$, Pyrazole (N)- $\mathrm{CH}_{3}$ ), 2.39 (s, 3H, Pyrazole- $\mathrm{CH}_{3}$ ). Anal. calcd for $\mathrm{C}_{10} \mathrm{H}_{10} \mathrm{Cl}_{2} \mathrm{~N}_{4} \mathrm{OS}$ : C 50.08, H 4.53, N 13.48; found C 50.12, H 4.52, N 13.22.

3.2.6 (E) 1,3-二甲基-5-芳氧基-1H-吡唑-4-甲醛 $O$-(2氯嘧啶-4)肜 $(B 1 \sim B 14)$ 的合成

在装有温度计的 $100 \mathrm{~mL}$ 四口瓶中, 加入(3 mmol) 5-芳氧基-1,3-二甲基- $1 H$-吡唑-4-甲醛基肜和 $0.828 \mathrm{~g} \mathrm{(6}$ $\mathrm{mmol}$ )碳酸钾, 再加入 $0.491 \mathrm{~g}(3.3 \mathrm{mmol}) 2,4$-二氯嘧啶 及 $40 \mathrm{~mL}$ 乙腈作为溶剂. 加热回流. TLC 跟踪反应至反 应完成. 过滤、浓缩, 得丙酮/石油醚 $\left(60 \sim 90{ }^{\circ} \mathrm{C}\right)(V / V=$ $1 / 5)$ 为洗脱剂进行柱层析得产品.

(E)-1,3-二甲基-5-苯氧基- $1 H$-吡唑-4-甲酫 $O$-(2-氯嘧 啶-4)肜(B1): 白色固体, 收率 77.1\%. m.p. $112 \sim 113{ }^{\circ} \mathrm{C}$; ${ }^{1} \mathrm{H}$ NMR $\left(\mathrm{CDCl}_{3}, 300 \mathrm{MHz}\right) \delta: 6.81 \sim 8.36(\mathrm{~m}, 8 \mathrm{H}, \mathrm{Ar}-\mathrm{H}$ \& Pyrimidine- $\mathrm{H} \& \mathrm{CH}=\mathrm{N}), 3.68(\mathrm{~s}, 3 \mathrm{H}$, Pyrazole $(\mathrm{N})-$ $\left.\mathrm{CH}_{3}\right), 2.46$ (s, 3H, Pyrazole- $\left.\mathrm{CH}_{3}\right)$; IR (KBr) v: 2980, 1721, 1561, 1423, 1285, 1191, 952, 853, $756 \mathrm{~cm}^{-1}$; MS (ESI) $m / z: 344.3[\mathrm{M}+\mathrm{H}]^{+}$. Anal. calcd for $\mathrm{C}_{16} \mathrm{H}_{14} \mathrm{ClN}_{5} \mathrm{O}_{2}: \mathrm{C}$ 55.90, H 4.10, N 20.37; found C 55.94, H 3.95, N 20.04.

(E)-1,3-二甲基-5-(2-甲苯氧基)- $1 H$-吡唑-4-甲醛 $O$ (2-氯嘧啶-4)肜(B2): 白色固体, 收率 65.0\%. m.p. 97 $98{ }^{\circ} \mathrm{C} ;{ }^{1} \mathrm{H}$ NMR $\left(\mathrm{CDCl}_{3}, 300 \mathrm{MHz}\right) \delta: 6.60 \sim 8.37(\mathrm{~m}, 7 \mathrm{H}$, $\mathrm{Ph}-\mathrm{H} \&$ Pyrimidine-H \& $\mathrm{CH}=\mathrm{N}), 3.69(\mathrm{~s}, 3 \mathrm{H}$, Pyrazole (N)- $\left.\mathrm{CH}_{3}\right), 2.45$ (s, 3H, Pyrazole- $\left.\mathrm{CH}_{3}\right), 2.40(\mathrm{~s}, 3 \mathrm{H}, \mathrm{Ph}-$ $\mathrm{CH}_{3}$ ); IR (KBr) v: 2943, 1559, 1425, 1294, 1144, 960, 849, $793,701 \mathrm{~cm}^{-1}$; MS (ESI) $\mathrm{m} / z: 358.9[\mathrm{M}+\mathrm{H}]^{+}$. Anal. calcd for $\mathrm{C}_{17} \mathrm{H}_{16} \mathrm{ClN}_{5} \mathrm{O}_{2}$ : C 57.07, $\mathrm{H}$ 4.51, N 19.57; found $\mathrm{C}$ 57.10, H 4.55, N 19.10 .

(E)-1,3-二甲基-5-(3-甲苯氧基)- $1 H$-吡唑-4-甲酫 $O$ (2-氯嘧啶-4)肜(B3): 黄色固体, 收率 76.0\%. m.p. 100
$102{ }^{\circ} \mathrm{C} ;{ }^{1} \mathrm{H}$ NMR $\left(\mathrm{CDCl}_{3}, 300 \mathrm{MHz}\right) \delta: 6.73 \sim 8.37(\mathrm{~m}$, $7 \mathrm{H}$, Ar-H \& Pyrimidine-H \& $\mathrm{CH}=\mathrm{N}), 3.67(\mathrm{~s}, 3 \mathrm{H}$, Pyrazole (N)- $\left.\mathrm{CH}_{3}\right), 2.46$ (s, 3H, Pyrazole- $\left.\mathrm{CH}_{3}\right), 2.35$ (s, $3 \mathrm{H}, \mathrm{Ar}-\mathrm{CH}_{3}$ ); IR (KBr) v: 2954, 1554, 1423, 1923, 1206, 952, 835, $698 \mathrm{~cm}^{-1}$. Anal. calcd for $\mathrm{C}_{17} \mathrm{H}_{16} \mathrm{ClN}_{5} \mathrm{O}_{2}: \mathrm{C}$ 57.07, H 4.51, N 19.57; found C 57.09, H 4.27, N 19.05.

(E)-1,3-二甲基-5-(4-甲苯氧基)- $1 H$-吡唑-4-甲醛 $O$ (2-氯嘧啶-4)肜(B4)：淡黄色固体，收率 78\%. m.p. 99 $101{ }^{\circ} \mathrm{C} ;{ }^{1} \mathrm{H}$ NMR $\left(\mathrm{CDCl}_{3}, 300 \mathrm{MHz}\right) \delta: 6.83 \sim 8.38(\mathrm{~m}$, $7 \mathrm{H}$, Ar-H \& Pyrimidine-H \& $\mathrm{CH}=\mathrm{N}), 3.67(\mathrm{~s}, 3 \mathrm{H}$, Pyrazole (N)- $\mathrm{CH}_{3}$ ), 2.46 (s, 3H, Pyrazole- $\left.\mathrm{CH}_{3}\right), 2.34$ (s, $\left.3 \mathrm{H}, \mathrm{Ar}-\mathrm{CH}_{3}\right)$; IR (KBr) v: 2927, 1555, 1423, 1346, 1299 , 960, 828, $708 \mathrm{~cm}^{-1}$. Anal. calcd for $\mathrm{C}_{17} \mathrm{H}_{16} \mathrm{ClN}_{5} \mathrm{O}_{2}$ : C 57.07, H 4.51, N 19.57; found C 57.42, H 4.58, N 18.22.

(E)-1,3-二甲基-5-(2-甲氧基苯氧基)- $1 H$-吡唑-4-甲 醛 $O-(2$-氯嘧啶-4)肜(B5): 白色晶体, 收率 59.3\%. m.p. $160 \sim 161{ }^{\circ} \mathrm{C} ;{ }^{1} \mathrm{H}$ NMR $\left(\mathrm{CDCl}_{3}, 300 \mathrm{MHz}\right) \delta: 6.80 \sim 8.40$ (m, 7H, Ar-H \& Pyrimidine-H \& $\mathrm{CH}=\mathrm{N}), 3.91(\mathrm{~s}, 3 \mathrm{H}$, Pyrazole (N)- $\left.\mathrm{CH}_{3}\right), 3.71\left(\mathrm{~s}, 3 \mathrm{H}, \mathrm{OCH}_{3}\right), 2.44(\mathrm{~s}, 3 \mathrm{H}$, Pyrazole- $\left.\mathrm{CH}_{3}\right)$; IR (KBr) v: 2968, 1607, 1551, 1424, 1343, 1299, 1200, 962, 840, 797, $714 \mathrm{~cm}^{-1}$. Anal. calcd for $\mathrm{C}_{17} \mathrm{H}_{16} \mathrm{ClN}_{5} \mathrm{O}_{3}$ : C 54.62, H 4.31, N 18.74; found C 54.70, H $4.49, \mathrm{~N} 18.68$.

(E)-1,3-二甲基-5-(3-硝基苯氧基)-1 $\mathrm{H}$-吡唑-4-甲醛 $O-(2$-氯嘧啶-4)肜(B6): 白色固体, 收率 69\%. m.p. 144 $146{ }^{\circ} \mathrm{C} ;{ }^{1} \mathrm{H}$ NMR $\left(\mathrm{CDCl}_{3}, 300 \mathrm{MHz}\right) \delta: 6.78 \sim 8.37(\mathrm{~m}$, $7 \mathrm{H}$, Ar-H \& Pyrimidine-H \& $\mathrm{CH}=\mathrm{N}), 3.72(\mathrm{~s}, 3 \mathrm{H}$, Pyrazole (N)- $\mathrm{CH}_{3}$ ), 2.47 (s, 3H, Pyrazole- $\mathrm{CH}_{3}$ ); IR ( $\mathrm{KBr}$ ) v: 3071, 2936, 1555, 1424, 1350, 1294, 1207, 960, 831, $717 \mathrm{~cm}^{-1}$. Anal. calcd for $\mathrm{C}_{16} \mathrm{H}_{13} \mathrm{ClN}_{6} \mathrm{O}_{4}$ : C 49.43, H 3.37, N 21.62; found C 49.39, H 3.45, N 21.40.

(E)-1,3-二甲基-5-(3-溴苯氧基)- $1 H$-吡唑-4-甲醛 $O$ (2-氯嘧啶-4)肜(B7): 白色固体, 收率 56.4\%. m.p. 120 $121{ }^{\circ} \mathrm{C} ;{ }^{1} \mathrm{H}$ NMR $\left(\mathrm{CDCl}_{3}, 300 \mathrm{MHz}\right) \delta: 6.83 \sim 8.38(\mathrm{~m}$, $7 \mathrm{H}$, Ar-H \& Pyrimidine-H \& $\mathrm{CH}=\mathrm{N}), 3.67(\mathrm{~s}, 3 \mathrm{H}$, Pyrazole (N)- $\mathrm{CH}_{3}$ ), 2.46 (s, 3H, Pyrazole- $\left.\mathrm{CH}_{3}\right), 2.34$ (s, $3 \mathrm{H}, \mathrm{Ar}-\mathrm{CH}_{3}$ ); IR (KBr) v: 3070, 2929, 1552, 1422, 1300, $1197,868,840,710 \mathrm{~cm}^{-1}$. Anal. calcd for $\mathrm{C}_{16} \mathrm{H}_{13} \mathrm{Br}-$ $\mathrm{ClN}_{5} \mathrm{O}_{2}$ : C 45.47, H 3.10, N 16.57; found C 45.65, H 3.09, $\mathrm{N} 16.57$.

(E)-1,3-二甲基-5-氯苯氧基- $1 H$-吡唑-4-甲醛 $O$-(2-氯 嘧啶-4)肜(B8): 白色固体, 收率 95\%. m.p. 137 139 ${ }^{\circ} \mathrm{C}$; ${ }^{1} \mathrm{H} \mathrm{NMR}\left(\mathrm{CDCl}_{3}, 300 \mathrm{MHz}\right) \delta: 6.79 \sim 8.38(\mathrm{~m}, 4 \mathrm{H}, \mathrm{Ar}-\mathrm{H}$ \& Pyrimidine- $\mathrm{H} \& \mathrm{CH}=\mathrm{N}), 3.68(\mathrm{~s}, 3 \mathrm{H}$, Pyrazole $(\mathrm{N})-$ $\mathrm{CH}_{3}$ ), 2.44 (s, 3H, Pyrazole- $\mathrm{CH}_{3}$ ); IR (KBr) v: 3071, 2929, 
1557, 1424, 1346, 1296, 959, 901, 854, 778, $714 \mathrm{~cm}^{-1}$. Anal. calcd for $\mathrm{C}_{16} \mathrm{H}_{13} \mathrm{Cl}_{2} \mathrm{~N}_{5} \mathrm{O}_{2}$ : C 50.81, H 3.46, $\mathrm{N} 18.52$; found $\mathrm{C} 51.06, \mathrm{H} 3.30, \mathrm{~N} 18.38$.

(E)-1,3-二甲基-5-(4-氯苯氧基)- $1 H$-吡唑-4-甲醛 $O$ (2-氯嘧啶-4)肜(B9): 白色固体, 收率 88\%. m.p. 125 $126{ }^{\circ} \mathrm{C} ;{ }^{1} \mathrm{H}$ NMR $\left(\mathrm{CDCl}_{3}, 300 \mathrm{MHz}\right) \delta: 6.78 \sim 8.38(\mathrm{~m}$, 7H, Ar-H \& Pyrimidine-H \& $\mathrm{CH}=\mathrm{N}), 3.67$ (s, 3H, Pyrazole (N)-CH ), 2.44 (s, 3H, Pyrazole- $\mathrm{CH}_{3}$ ). IR (KBr) v: 3093, 2943, 1719, 1557, 1427, 1292, 1200, 1090, 957, $834,714 \mathrm{~cm}^{-1}$; MS (ESI) $\mathrm{m} / z: 378.1[\mathrm{M}+\mathrm{H}]^{+}$. Anal. calcd for $\mathrm{C}_{16} \mathrm{H}_{13} \mathrm{Cl}_{2} \mathrm{~N}_{5} \mathrm{O}_{2}$ : C 50.81, H 3.46, N 18.52; found $\mathrm{C}$ 50.90, H 3.43, N 18.62 .

(E)-1,3-二甲基-5-(2,4-二氯苯氧基)-1H-吡唑-4-甲醛 O-(2-氯嘧啶-4)肟(B10): 白色固体, 收率 49.0\%. m.p. $124 \sim 126{ }^{\circ} \mathrm{C} ;{ }^{1} \mathrm{H}$ NMR $\left(\mathrm{CDCl}_{3}, 300 \mathrm{MHz}\right) \delta: 6.68 \sim 8.41$ (m, 6H, Ar-H \& Pyrimidine-H \& $\mathrm{CH}=\mathrm{N}), 3.70(\mathrm{~s}, 3 \mathrm{H}$, Pyrazole (N)-CH ), 2.43 (s, 3H, Pyrazole- $\mathrm{CH}_{3}$ ); IR (KBr) v: 3074, 2929, 1554, 1223, 1344, 1298, 1219, 959, 709 $\mathrm{cm}^{-1}$. Anal. calcd for $\mathrm{C}_{16} \mathrm{H}_{12} \mathrm{Cl}_{3} \mathrm{~N}_{5} \mathrm{O}_{2}: \mathrm{C} 46.57, \mathrm{H} 2.93, \mathrm{~N}$ 16.97; found C 46.35, H 3.09, N 17.08.

(E)-1,3-二甲基-5-(3,5-二甲苯氧基)- $1 H$-吡唑-4-甲醛 O-(2-氯嘧啶-4)肜(B11): 白色固体, 收率 77\%. m.p. $102 \sim 103{ }^{\circ} \mathrm{C} ;{ }^{1} \mathrm{H}$ NMR $\left(\mathrm{CDCl}_{3}, 300 \mathrm{MHz}\right) \delta: 6.55 \sim 8.38$ $(\mathrm{m}, 6 \mathrm{H}, \mathrm{Ar}-\mathrm{H} \&$ Pyrimidine-H \& $\mathrm{CH}=\mathrm{N}), 3.66(\mathrm{~s}, 3 \mathrm{H}$, Pyrazole (N)- $\mathrm{CH}_{3}$ ), 2.47 (s, 3H, Pyrazole- $\left.\mathrm{CH}_{3}\right), 2.29$ (s, $6 \mathrm{H}, \mathrm{Ar}-\mathrm{CH}_{3}$ ); IR (KBr) v:2926, 1555, 1424, 1297, 1204, 966, 834, $715 \mathrm{~cm}^{-1}$. Anal. calcd for $\mathrm{C}_{18} \mathrm{H}_{18} \mathrm{ClN}_{5} \mathrm{O}_{2}: \mathrm{C}$ 58.14, H 4.88, N 18.84; found C 58.19, H 5.01, N 18.79.

(E)-1,3-二甲基-5-(2,4-二甲苯氧基)- $1 H$-吡唑-4-甲醛 O-(2-氯嘧啶-4)肜(B12): 白色固体, 收率 80.1\%. m.p. $92 \sim 93{ }^{\circ} \mathrm{C} ;{ }^{1} \mathrm{H} \mathrm{NMR}\left(\mathrm{CDCl}_{3}, 300 \mathrm{MHz}\right) \delta: 6.51 \sim 8.38(\mathrm{~m}$, $6 \mathrm{H}$, Ar-H \& Pyrimidine-H \& $\mathrm{CH}=\mathrm{N}), 3.68(\mathrm{~s}, 3 \mathrm{H}$, Pyrazole (N)- $\mathrm{CH}_{3}$ ), 2.45 (s, 3H, Pyrazole- $\left.\mathrm{CH}_{3}\right), 2.34$ (s, $3 \mathrm{H}, \mathrm{Ar}-\mathrm{CH}_{3}$ ), 2.30 (s, 3H, $\mathrm{Ar}-\mathrm{CH}_{3}$ ); IR (KBr) v: 2926, 1553, 1420, 1298, 1197, 1119, 957, 817, $709 \mathrm{~cm}^{-1}$; MS (ESI) $m / z: 372.1[\mathrm{M}+\mathrm{H}]^{+}$. Anal. calcd for $\mathrm{C}_{18} \mathrm{H}_{18} \mathrm{ClN}_{5} \mathrm{O}_{2}$ : C 58.14, H 4.88, N 18.84; found C 58.33, H 4.66, N 18.72.

(E)-1,3-二甲基-5-(3-三氟甲基苯氧基)- $1 H$-吡唑-4甲醛 $O-(2$-氯嘧啶-4)肜(B13): 白色晶体, 收率 73\%. m.p. $114 \sim 116{ }^{\circ} \mathrm{C} ;{ }^{1} \mathrm{H}$ NMR $\left(\mathrm{CDCl}_{3}, 300 \mathrm{MHz}\right) \delta: 6.66 \sim 8.34$ $(\mathrm{m}, 7 \mathrm{H}, \mathrm{Ar}-\mathrm{H} \&$ Pyrimidine-H \& $\mathrm{CH}=\mathrm{N}), 3.71(\mathrm{~s}, 3 \mathrm{H}$, Pyrazole (N)- $\mathrm{CH}_{3}$ ), 2.46 (s, 3H, Pyrazole- $\mathrm{CH}_{3}$ ); IR (KBr) v: 3067, 2929, 1555, 1423, 1326, 1170, 1123, 971, 708 $\mathrm{cm}^{-1}$. Anal. calcd for $\mathrm{C}_{17} \mathrm{H}_{13} \mathrm{ClF}_{3} \mathrm{~N}_{5} \mathrm{O}_{2}$ : C 49.59, $\mathrm{H} 3.18, \mathrm{~N}$ 17.01; found C 49.40, H 3.09, N 16.84 .
(E)-1,3-二甲基-5-(4-甲氧羰基苯氧基)- $1 H$-吡唑-4甲醛 $O-(2$-氯嘧啶-4)肜(B14): 白色固体, 收率 $62 \%$. m.p. $151 \sim 152{ }^{\circ} \mathrm{C} ;{ }^{1} \mathrm{H}$ NMR $\left(\mathrm{CDCl}_{3}, 300 \mathrm{MHz}\right) \delta: 6.71 \sim 8.32$ $(\mathrm{m}, 7 \mathrm{H}, \mathrm{Ar}-\mathrm{H} \&$ Pyrimidine-H \& $\mathrm{CH}=\mathrm{N}), 3.09$ (s, 3H, $\left.\mathrm{CO}_{2} \mathrm{CH}_{3}\right), 3.67\left(\mathrm{~s}, 3 \mathrm{H}\right.$, Pyrazole (N)- $\left.\mathrm{CH}_{3}\right), 2.44(\mathrm{~s}, 3 \mathrm{H}$, Pyrazole- $\mathrm{CH}_{3}$ ); IR (KBr) v: 3053, 2950, 1713, 1554, 1424, 1294, 969, $710 \mathrm{~cm}^{-1}$. Anal. calcd for $\mathrm{C}_{18} \mathrm{H}_{16} \mathrm{ClN}_{5} \mathrm{O}_{4}: \mathrm{C}$ 53.81, H 4.01, N 17.43; found C 53.80, H 3.91, N 17.47.

\section{3 生物活性测试}

\subsection{1 杀虫测试方法}

杀虫活性测试所用昆虫分别为: 粘虫(Mythimna separata Walker)、尖音库蚊淡色亚种 (Culex pipiens pallens)、蚕豆蚜(Aphis laburni Kaltenbach)以及朱砂叶 螨, (Tetranychus cinnabarinus).

杀虫活性测试试验方法分别如下所述:

蚊幼虫: 用浸液法, 将 4 龄幼虫放入一定浓度 $(5$ $\mathrm{mg} \cdot \mathrm{L}^{-1}$ ) 的药液(水)中. $24 \mathrm{~h}$ 检查蚊幼虫死亡率结果.

蚜虫: 称取 $12 \mathrm{mg}$ 药样于 $100 \mathrm{~mL}$ 烧杯中, 加 $0.5 \mathrm{~mL}$ $\mathrm{DMF}$ (或其它适合溶剂), 最多不超过 $1 \mathrm{~mL}$; 加乳化剂 Sorpol 560(或其它适合乳化剂)1 滴，用玻璃棒充分搅拌, 使其互相溶解，再加适量蒸馏水后，再次充分搅拌，配 成 $200 \mathrm{mg} \cdot \mathrm{L}^{-1}$ 的药液. 将带有若虫的植株在上述药液中 浸渍 $2 \sim 3 \mathrm{~s}$, 甩掉多余药液, 将植株扦在泡沫板上, 加 盖煤油灯灯罩(灯罩上口用纱布、橡皮圈封口), 并将试 验置于处理室内, $24 \mathrm{~h}$ 检查结果.

粘虫: 浸叶法, 用玉米叶浸渍于丙酮配制的药液中 $\left(500 \mathrm{mg} \cdot \mathrm{L}^{-1}\right)$, 待药液后接入 4 龄幼虫, 主要为胃毒、触 杀作用, 同时观察幼虫取食现象, $24 \mathrm{~h}$ 检查死亡率.

螨虫: 称取 $12 \mathrm{mg}$ 药样于 $100 \mathrm{~mL}$ 烧杯中, 加 $1 \mathrm{~mL}$ 丙酮(或其它适合溶剂). 加乳化剂 Sorpol 560(或其它适 合乳化剂) 1 滴, 用玻璃棒充分搅拌, 使其互相溶解, 再 加蒸馏水后, 再次充分搅拌, 配成 $200 \mathrm{mg} \cdot \mathrm{L}^{-1}$ 的药液. 将带有叶螨的植株在上述药液中浸渍 $5 \mathrm{~s}$, 轻轻抖动, 去 掉多余的药液, 将植株插入带有方块玻璃盖的水培缸 中, 玻璃盖四周涂以羊毛脂, 防止螨虫逃走, 把试验置 于处理室内, 24 或 $48 \mathrm{~h}$ 检查结果.

\section{3 .2 杀菌活性测试方法}

将一定量药剂溶解在适量二甲基甲酰胺(DMF)内, 然后用乳化水稀释至 $50 \mu \mathrm{g} \cdot \mathrm{mL}^{-1}$. 蕃茄早疫病菌 (Alternaria solani)、花生褐斑病菌(Cercospora arachidicola)、芦笋茎枯病菌(Phomopsisasp-aragi)、小麦赤霉 病菌(Gibberella zeae)和苹果轮纹病菌(Physalospor piricola)为供试病原菌, 供试菌均为本所保存菌株, 采用 PDA 培养基, 经活化后备用.

采用菌体生长速率测定法(mycelium growth rate 
test). 具体过程是: 在无菌条件下各吸取 $1 \mathrm{~mL}$ 药液注入 培养血内, 加入 $9 \mathrm{~mL}$ PDA 培养基, 摇匀后制成 $50 \mu \mathrm{g}$ ・ $\mathrm{mL}^{-1}$ 含药平板, 以添加 $1 \mathrm{~mL}$ 灭菌水的平板做空白对照. 用直径 $4 \mathrm{~mm}$ 的打孔器沿菌丝外缘切取菌盘, 移至含药 平板上, 每处理重复三次. 将培养血放在 $(24 \pm 1){ }^{\circ} \mathrm{C}$ 恒 温培养箱内培养. $72 \mathrm{~h}$ 后调查各处理菌盘扩展直径, 求 平均值, 与空白对照比较计算相对抑菌率.

相对抑菌率 $(\%)=($ 对照组菌盘扩展平均直径一处理

组菌盘扩展平均直径) / 对照组菌盘扩展平均直径 $\times$ $100 \%$

致谢 感谢南开大学元素有机化学研究所生测组同事 的辛勤劳动!

辅助材料(Supporting Information) 目标化合物 A1 $\mathbf{A 2 1}$ 和 B1 $\sim$ B14 的 ${ }^{1} \mathrm{H}$ NMR 数据. 这些材料可以免费从 本刊网站(http://sioc-journal.cn/)上下载.

\section{References}

[1] Park, H. J.; Lee, K.; Park, S. J.; Ahn, B.; Lee, J. C.; Cho, H. Y.; Lee, K. I. Bioorg. Med. Chem. Lett. 2005, 15(13), 3307.

[2] Drabek, J. DE 4200742, 1992 [Chem. Abstr. 1992, 117, 171437].

[3] Zhao, P. L.; Wang, L.; Zhu, X. L.; Huang, X. Q.; Zhan, C. G.; Wu, J. W.; Yang, G. F. J. Am. Chem. Soc. 2010, 132, 185.

[4] Obata, T.; Fujii, K.; Fukuda, Y.; Tsutsumiuchi, K. JP 03240775, 1991 [Chem. Abstr. 1991, 116, 101166].
[5] Hamaguchi, H.; Kajihara, O.; Katoh, M. J. Pestic. Sci. 1995, 20, 173.

[6] Zhang, S.; Zeng, X. N.; Luo, Y. Plat Protection 2004, 30, 11 (in Chinese).

(张帅，曾金金年，骆悦，植物保护, 2004, 30, 11.)

[7] Peter, L. Biochim. Biophys. Acta 1998, 1364, 287.

[8] Yang, H. Z.; Li, Q. Agrochemicals 2007, 46, 81 (in Chinese). (杨会芝, 李庆, 农药, 2007, 46, 81.)

[9] Fujimoto, H.; Mikitani, K.; Meki, N.; Imahase, T.; Nishida, K.; Fujimoto, H.; Mikitani, K.; Takano, H.; Ogasawara, Y.; Tamaki, M. EP 390498, 1990 [Chem. Abstr. 1991, 114, 101986].

[10] Obata, T.; Fujii, K. EP 391685, 1990 [Chem. Abstr. 1991, 114, 101987].

[11] Dai, H.; Yu, H. B.; Liu, J. B.; Qin, X.; Wang, T. T.; Zhang, X.; Qin, Z. F.; Fang, J. X. Chin. J. Org. Chem. 2013, 33, 1104 (in Chinese). (戴红, 于海波, 刘建兵, 秦雪, 王婷婷, 张欣, 秦振芳, 方建新, 有机化学, 2013, 33, 1104.)

[12] Fu, C. R.; Pei, J.; Ning, Y.; Liu, M.; Shan, P. C.; Liu, J.; Li, Y. Q.; Hu, F. Z.; Zhu, Y. Q.; Yang, H. Z.; Zou, X. M. Pest Manage. Sci. 2014, 70, 1207.

[13] Kagabu, S. Rev. Toxicol. 1997, 1, 75.

[14] Jia, J. C.; Yuan, J. X.; Fan, Z. J. Agrochemicals 2007, 46, 228 (in Chinese). (贾俊超, 苑建勋, 范志金, 农药, 2007, 46, 228.)

[15] Yokota, T.; Mikata, K.; Nagasaki, H.; Ohta, K. J. Agric. Food Chem. 2003, 51, 7066.

[16] Wu, Q.; Zhang, Q.; Yang, J. C.; Liu, C. L. Agrochemicals 2010, 49, 2 (in Chinese). (伍强, 张茜, 杨吉春, 刘长令, 农药, 2010, 49, 2.)

[17] Qu, F. J.; Lu, Q. S. J. Huaihai Inst. Technol. 2003, 12, 45 (in Chinese).

(曲富军, 陆庆松, 淮海工学院学报, 2003, 12, 45.)

(Cheng, F.) 